\title{
Laparoscopic sleeve gastrectomy without over-sewing of the staple line is effective and safe
}

\author{
Mojmir Kasalicky ${ }^{1,2}$, Radek Dolezel ${ }^{1}$, Eva Vernerova ${ }^{1}$, Martin Haluzik $^{3}$ \\ ${ }^{1} 2^{\text {nd }}$ Medical Faculty of Charles University and Central Military Hospital, Prague, Czech Republic \\ ${ }^{2}$ Faculty of Health Care and Social Work, Trnava University, Trnava, Slovak Republic \\ ${ }^{3} 3^{\text {rd }}$ Internal Department General Faculty Hospital, $1^{\text {st }}$ Medical Faculty of Charles University, Prague, Czech Republic
}

Videosurgery Miininv (e-pub, ahead of print) DOI: $10.5114 /$ wiitm.2014.40387

\begin{abstract}
Introduction: Laparoscopic sleeve gastrectomy (LSG) is a bariatric procedure with very good long-term weight-reducing and metabolic effects.

Aim: Here we report 6 years' experience with LSG performed in morbidly obese patients by one surgical team focusing on the impact of the degree of sleeve restriction and safety of the procedure without over-sewing the staple line. Material and methods: From 2006 to 2012, 207 morbid obese patients with average age of 43.4 years and average body mass index $44.9 \mathrm{~kg} / \mathrm{m}^{2}$ underwent LSG without over-sewing the staple line. The complete 5-and 3-year follow-up is recorded in 59 and 117 patients with prospective data collection at 3, 6, 9, 12, 18, 24, 36, 42 and 60 months after LSG. Group 1 patients operated in 2006-2008 had smaller sleeve restriction. Group 2 patients operated in 2009-2012 had major sleeve restriction. All procedures were performed without over-sewing of the staple line.

Results: The average \%EBMIL (excess body mass index loss) in group 1 patients with minor sleeve restriction reached $54.1 \%$ and average \%EWL (excess weight loss) was 50.8\% while in group 2 with major sleeve restriction the average $\% E B M I L$ reached $69.7 \%$ and average \%EWL was 66.8\%. Final weight reduction was significantly higher in group 2 patients compared to group 1 patients with smaller sleeve restriction. Out of 49 patients with preoperatively diagnosed T2DM (type 2 diabetes mellitus) was completely resolved in $70.8 \%$. Pre-operatively diagnosed hypertension normalized in $64.2 \%$, improved in $23.2 \%$, and remained unchanged in $12.6 \%$ of patients.

Conclusions: Carefully performed LSG without over-sewing the staple line is feasible and safe. A better weight-reducing effect was present in patients with major sleeve restriction.
\end{abstract}

Key words: morbid obesity, laparoscopic sleeve gastrectomy, diabetes, staple line.

\section{Introduction}

Morbid obesity represents the most common multifactorial metabolic disorder and a major health problem worldwide. Obesity is frequently accompanied by numerous related pathologies including type 2 diabetes (T2DM), hypertension, steatohepatitis, pulmonary dysfunction, cardiovascular disease and malignant tumours commonly referred to as metabolic syndrome resulting in markedly increased cardiovascular risk and mortality of affected patients [1]. Myocardial infarction is three times and T2DM is even five times more frequent in obese patients with metabolic syndrome compared to the lean population [2]. Bariatric/ metabolic (BM) surgery is a rapidly developing field of the miniinvasive laparoscopic surgery with powerful treatment effects not only on obesity but also on accompanying metabolic pathologies $[1,3]$.

\section{Address for correspondence}

Prof. Mojmir Kasalicky MD, PhD, $2^{\text {nd }}$ Medical Faculty of Charles University and Central Military Hospital, U Vojenske nemocnice 1200 ,

16902 Prague 6, Czech Republic, phone: +42 0973202 901, e-mail: mojmir.kasalicky@uvn.cz 
Current surgical bariatric/metabolic methods can be roughly divided into restrictive procedures, malabsorptive procedures or a combination of methods. Laparoscopic sleeve gastrectomy (LSG) has become a very popular and useful bariatric procedure, which shows very promising and satisfactory results even when used as a single procedure.

Sleeve gastrectomy was introduced into the spectrum of bariatric surgery by Hess, who performed sleeve gastrectomy as part of duodenal switch (DS) for the first time in 1988, and Johnson presented a similar method in 1993 as the Magenstrasse and Mill operation [2, 4-7]. Gagner performed laparoscopic sleeve gastrectomy in 1999 and published the first results of this method using it as a first-step procedure in super-obese patients before laparoscopic duodenal switch or biliopancreatic diversion [5, 8]. During the last $5 / 6$ years several mid-term studies revealed that $\mathrm{LSG}$ might be even used as a single restrictive bariatric method for morbid obesity (MO) with body mass index $(\mathrm{BMI}) \leq 40 \mathrm{~kg} / \mathrm{m}^{2}$, as well as in super-obese patients with $\mathrm{BMI} \leq 50 / 60 \mathrm{~kg} / \mathrm{m}^{2}$, with very satisfactory weight loss and improvement of metabolic disorders such as T2DM, dyslipidaemia and others [9-11].

The indication for laparoscopic sleeve gastrectomy was usually morbid obesity (BMI $\left.>40 \mathrm{~kg} / \mathrm{m}^{2}\right)$ in patients who have failed conservative therapy and/ or $\mathrm{BMI}>35-40 \mathrm{~kg} / \mathrm{m}^{2}$ with serious obesity-related complications [8, 12].

The results from some studies show that LSG is associated with a high rate of resolution of T2DM and improvement of hypertension, hyperlipidaemia, cardio-respiratory disease, obesity-related severe psychological problems, sleep apnoea and others [8, 11, 13]. Weight reduction and resolution of these comorbidities after LSG have been similar to the effect of gastric bypass and larger than after adjustable gastric banding. Significant long-term nutritional deficiencies have not been described with this procedure, making LSG a relatively simple bariatric method with very low frequency of complications if performed by an experienced surgical team $[11,13]$. The effects of LSG are based on three principles: reduction of the gastric volume capacity by resection of the gastric body, fundus and part of the antrum; long-term decrease of the orexigenic hormone ghrelin concentrations; and acceleration of gastric emptying [14-16].

Some variations exist as far as the LSG operation technique is concerned which may potentially affect its short- and long-term outcomes. The resection of part of the stomach is done along the calibrating bougie or orogastric tube with diameter from $32 \mathrm{Fr}$ to $50 \mathrm{Fr}$. It should be mentioned that a smaller diameter (32-42 Fr) of calibrating bougie seems to have a better effect on postoperative weight loss in LSG patients [8, 17-19]. Bariatric surgeons frequently use over-sewing running or interrupted sutures (non-absorbable or absorbable) to reinforce the staple line after gastric sleeve resection, in order to lower the risks of consequent haemorrhages and/or leaks $[20,21]$.

\section{Aim}

Here we report our 6 years of experience with 207 consecutive LSG performed in morbidly obese patients by one surgical team. The procedures were done without using reinforcing sutures over the staple line. Specifically, we analyse the safety of the LSG procedure without over-sewing of the staple line and the impact of the degree of sleeve restriction on long-term weight-reducing effects. Our data suggest that this technique represents a safe approach with very satisfactory short- and long-term results.

\section{Material and methods}

Our study includes 207 morbidly obese patients who underwent LSG from 2006 to 2012 and data were collected prospectively. All patients were referred for the operation after the consensus of a multidisciplinary team, which consisted of the surgeon, an internal specialist in obesitology/diabetology and a psychologist, and in agreement with the Interdisciplinary European Guidelines for Surgery of Severe Obesity [12]. Before the operation, all patients were required to undergo the entire range of blood laboratory tests, electrocardiogram, pulmonary function test, gastroscopy, upper gastrointestinal X-ray, abdominal ultrasound, psychological examination and detailed education about the sleeve gastrectomy technique and its consequences.

Our cohort of 207 morbid obese patients included 48 men and 159 women with average age of 43.4 years (range 19-65), average height of $169.3 \mathrm{~cm}$ (range 151-192), average weight of $128.7 \mathrm{~kg}$ (range 96-184) and average BMI 44.9 (range 34.1-71.9) (Table I). There were no statistically significant differences in demographics or comorbidities between men and woman. The complete 5-year follow-up is 
recorded in 61 patients and complete 3-year follow-up is recorded in 114 patients. During preoperative investigation, obesity-related T2DM was detected in $49(23.6 \%)$ cases with average fasting plasma glucose $9.3 \mathrm{mmol} / \mathrm{l}$ (range $7.2-14.1 \mathrm{mmol} / \mathrm{l}$ ). Type 2 diabetes mellitus has been treated for an average duration of 4.7 years (range $1.5-7.5$ ) before surgery. In 109 (52.7\%) cases systolic/diastolic hypertension was detected with average blood pressure of $149 /$ 96 mm Hg (range 135-165/90-105 mm Hg). Ninety-seven (89\%) patients were on anti-hypertension medication.

Patients were divided into two groups by date and type of surgery. Group 1 patients with minor sleeve restriction were operated on in 2006-2008; group 2 patients with major sleeve restriction were operated on in 2009-2012. All data including patient's weight, BMI, development of T2DM, hypertension and incidence of complications were recorded prospectively at 3, 6, 9, 12, 18, 24, 36, 42 and 60 months after LSG.

\section{Statistical analysis}

The observed results were evaluated by descriptive statistics methods and Mann-Whitney rank test.

\section{Surgical technique}

Our technique was similar to techniques described by others, except that LSG was performed without over-sewing of the stomach staple line in all cases. Under general anaesthesia, the patient was placed in lithotomic and in reverse Trendelenburg position with sequential compression of lower limbs, in addition to standardized prevention of deep vein thrombosis (DVT) with low molecular weight heparin. The operating surgeon was positioned between the patient's legs. A capnoperitoneum was created with $\mathrm{CO}_{2}$ through the use of a Veress needle with intra-abdominal pressure not exceeding $15 \mathrm{~mm} \mathrm{Hg}$. Five trocars were used (two $12.5 \mathrm{~mm}$ and three 5 $\mathrm{mm}$ ). The port for optics was positioned slightly to the patient's left side, above the umbilicus. In patient group 1 the dissection of the greater gastric curvature started at the point measured $6 \mathrm{~cm}$ from the pylorus in the period from 2006 to 2008 and proceeded upwards until the angle of His. In this group a $42 \mathrm{Fr}$ calibrating bougie was then inserted by the anaesthesiologist into the stomach and was directed closely along the lesser curvature towards the pylorus. In patient group 2 the distance of the staple
Table I. Patients' demographics

\begin{tabular}{|lc|}
\hline Parameter & Results \\
\hline$N$ & 207 \\
\hline Age [years] & $43.4(19-65)$ \\
\hline Women & 159 \\
\hline Men & 48 \\
\hline Height $[\mathrm{cm}]$ & $169.3(151-192)$ \\
\hline Weight $[\mathrm{kg}]$ & $128.7(96-184)$ \\
\hline BMl $\left[\mathrm{kg} / \mathrm{m}^{2}\right]$ & $44.9(34.1-71.9)$ \\
\hline
\end{tabular}

line's beginning from the pylorus was $3-4 \mathrm{~cm}$ and a $36 \mathrm{Fr}$ calibrating bougie was used to obtain better restriction with smaller residual volume of the sleeve in the period from 2009 to 2012. The resection line ended $1.0-1.5 \mathrm{~cm}$ from the angle of His to preserve the lower sphincter of the oesophagus for prevention of heartburn.

For tissue and vessel dissection an ultrasonic dissection device was used (Harmonic Scalpe ${ }^{\mathrm{TM}}$ Ethicon Endo-Surgery). Dissection of adhesions between the back of the stomach wall and pancreas was carried out, followed by very careful resection of the gastric wall. In the majority of cases a linear stapler (Echelon60, Ethicon Endo-Surgery) with blue cartridges was used. We recommend holding on for approximately $60 \mathrm{~s}$ after stapler closure and before firing it, as well as before removing the stapler. In our experience this allows more accurate closure of staplers at the resection line, which may contribute to lower rates of staple-line bleeding. At the end of this phase of the procedure the calibrating bougie was removed. If bleeding occurred at the staple line area, one or several interrupted stitches were sufficient to control the bleeding. Thereafter a leak test with methylene blue was used to detect the integrity of the staple line occasionally at the beginning of the method.

In none of our patients did we over-sew the gastric resection/staple line. Sporadically, only in case of a small diffuse blood leak was the resection/staple line covered with $100 \%$ oxidized cellulose (Surgicel $^{\mathrm{TM}}$, Ethicon). However, we suppose that it was neither sutured nor stapled to the gastric tissue, and thus it did not act as a staple line reinforcement. The resected stomach portion was extracted from the abdominal cavity through the dilatated $12.5 \mathrm{~mm}$ trocar incision. Approximately the first 60 procedures 
were ended with the suction drain placed alongside the staple line; however, from 2009 the drain was used rather infrequently. X-ray upper Gl series with Gastrografin ${ }^{\circledR}$ was carried out in all patients on the second/third postoperative day. Proton pump inhibitors (PPI) and prokinetics drugs were administered intravenously during the first $48 \mathrm{~h}$ after the procedure. Antibiotics were administered for the first $72 \mathrm{~h}$ at the beginning, but from 2009 antibiotics were administered just 60 min before the procedure, with an identical effect. Patients were mobilized within 6-8 h after the operation. During the first 2 weeks a low-sodium semi-liquid diet was advised with subsequent low-fat and low-carbohydrate diet use. The adequate intake of liquids was strongly recommended.

\section{Results}

Mean operating time was 105 min (75-170) in group 1 and $83 \min$ (65-125) in group 2. In group 1 we had to switch in one case from laparoscopy to open surgery because of bleeding from the spleen that was injured during dissection and in the same group 1 there was only one patient with an early serious postoperative complication - intra-abdominal bleeding from the resection line requiring laparoscopic revision within $4 \mathrm{~h}$ after surgery. During this urgent revisional surgery, the bleeding point in the residual antrum was over-sewn with a single interrupted resorbable stitch.

Early complications - wound infection at the site of the drainage and/or specimen extraction occurred in 4 patients. Intensive nausea lasting for several hours after the procedure was recorded in more than $50 \%$ of our patients after LSG. The nausea could be managed with intravenous application of antiemetic drugs.

Another postoperative complication, which occurred in group 12 weeks after LSG, was the leak

Table II. Effect of LSG on weight loss in group 1 and group 2

\begin{tabular}{|lc|}
\hline \multicolumn{2}{|l|}{ Group 1: LSG 2006-2008 $(n=59)$} \\
\hline \%EBMIL & $54.1(19.3-92.9)$ \\
\hline \%EWL & $50.8(18.7-97.1)$ \\
\hline Group 2: LSG 2009-2011 $(n=117)$ \\
\hline \%EBMIL & $69.7(24.2-120.9)$ \\
\hline$\%$ EWL & $66.8(22.5-113.8)$ \\
\hline
\end{tabular}

Mann-Whitney test: $p=0.0495$ from the staple line in 2006 when gold cartridges were used. This perigastric abscess had to be treated by CT-controlled drainage.

Mean postoperative stay was 4.2 days (range $3-7$ ). No peri- or postoperative mortality was encountered.

From 2006 to 2012, 207 LSG were done. Data were recorded prospectively at 3, 6, 9, 12, 18, 24, 36, 42 and 60 months in patients after LSG. Thirty-one patients $(15.1 \%)$ were not included in the analysis due to incomplete data (exclusion criteria were wrong patient's compliance, lost to follow-up or due to short time after the operation - for less than 6 months). Nevertheless, serious complications such as bleeding, leak from the staple line or stenosis of the sleeve did not occur in those excluded patients. The complete 3-year follow-up is recorded in 176 patients and from this group a complete 5-year follow-up is recorded in 61 patients. We discovered that the weight loss finished during $23 \pm 4$ months in all of our patients. On the basis of this fact we decided to evaluate acquired data (\%EBMIL, \%EWL, T2DM, etc.) over the period of 3 years so as to have the same criteria in both groups. In the group 1 patients (period 2006-2008, $n=59$ ) with minor sleeve restriction, the average \%EBMIL was $54.1 \%$ (range 19.3-92.9\%) and average \%EWL was 50.8\% (range 18.7-97.1\%). In the group 2 patients (period 2009$2012, n=117$ ) with major sleeve restriction the average \%EBMIL was $69.7 \%$ (range $24.2-120.9 \%$ ) and average \%EWL was 66.8\% (range 22.5-113.8\%) (Table II). Morbidly obese patients after LSG in group 2 with major sleeve restriction (period 2009-2012) had been losing weight easily and achieved better final weight reduction than patients in group 1 with minor sleeve restriction (period 2006-2008) (MannWhitney test: $p=0.0495$ ).

As expected, LSG substantially improved or resolved several obesity-related co-morbidities. Out of the pre-operatively diagnosed 49 T2DM patients, 35 of them were on oral anti-diabetic medications (OAD) and 14 on combined therapy with insulin and OAD. In this postoperative period their diabetes completely resolved in 34 cases $(71.4 \%)$ and improved in other patients with T2DM after surgery. Pre-operatively diagnosed hypertension normalized in $64.2 \%$, improved in $23.2 \%$ and remained unchanged in $12.6 \%$ out of 95 hypertonic patients with complete 3-year follow-up (Table III).

During 3 years of follow-up $31 \%$ of patients experienced mild heartburn after surgery which disap- 
peared within 6-9 months. But in $14 \%$ of patients heartburn persisted in long-term follow-up after surgery. They have to be on PPI. None of our patients have developed dumping syndrome, peptic ulcer, diarrhoea, anaemia or hypovitaminosis so far.

\section{Discussion}

In our study, we analyzed the safety of the LSG procedure without over-sewing of the staple line and the impact of the degree of sleeve restriction on long-term weight-reducing effects.

Laparoscopic sleeve gastrectomy has become a popular bariatric procedure with a very good effect as far as long-term weight loss and improvement of metabolic disorders are concerned. Our surgical team has experience with this continuously more frequent bariatric procedure since 2006. In our surgical department, we laparoscopically perform gastric bandings and gastric vertical plications, but LSG represents the most frequently performed procedure. In the case of non-satisfactory weight loss and metabolic improvement during 1 year after LSG we perform a duodenojejunal bypass sleeve gastrectomy as a second step operation.

The current clinical experience shows that sleeve gastrectomy can be used as a single bariatric/metabolic procedure because of its restrictive (gastric resection) and hormonal (ghrelin) mode of action combined with faster gastric emptying [14, 15, 22]. The very good metabolic effect of sleeve gastrectomy (SG) of resolving or improving T2DM within a short timeframe after the procedure can be explained by the hindgut hypothesis. Poorly pre-digested food, which is promptly transiting from the sleeve through the oral jejunum to the distal bowel, improves glucose metabolism by stimulating intestinal cells to secrete glucagon-like peptide 1 (GLP-1) and/or other incretins. According to some other studies, insulin secretion is also improved followed by improvement of the glucose tolerance [23-25]. Basso speculates about the gastric hypothesis of the LSG mechanism of action: decreased $\mathrm{HCl}$ production induced by SG may act on the innervated antrum to produce gastrin-releasing peptide responsible for GLP-1 early-phase secretion [26].

An increasing number of authors confirm that LSG is feasible with an adequate learning curve and low morbidity and mortality. Advantages of this approach include the lack of nutrient malabsorption or
Table III. Effect of LSG on improving/resolving T2DM and hypertension after 3 years

\begin{tabular}{|llcc|}
\hline & Resolved & Improved & Unchanged \\
\hline T2DM $(n=49)$ & $34(71.4 \%)$ & $15(28.6 \%)$ & 0 \\
\hline HT $(n=95)$ & $61(64.2 \%)$ & $22(23.2 \%)$ & $12(12.6 \%)$ \\
\hline T2DM - type 2 diabetes mellitus, HT - hypertension &
\end{tabular}

dumping syndrome. Furthermore, there are no problems with postoperative endoscopic examination of the whole residual stomach or cholangiography [13].

During them last few years some surgical technique modifications have been introduced, but the majority of authors are presently using a calibrating bougie from $32 \mathrm{Fr}$ to $38 \mathrm{Fr}$ and beginning the resection line $3-4 \mathrm{~cm}$ from the pylorus to obtain better restriction. In our study, we have compared two different sizes of sleeve calibration: $42 \mathrm{Fr}$ orogastric tube in group 1 patients operated on in 2006-2008 vs. $36 \mathrm{Fr}$ orogastric tube for sleeve calibration in group 2 patients operated on in 2009-2012. We show that the latter approach results in significantly higher final weight reduction compared to smaller sleeve restriction. In our experience better restriction can be achieved by beginning resection $3-4 \mathrm{~cm}$ from the pylorus for lowering residual sleeve volume without downgrade of the antrum evacuation.

Another important focus of this study was to assess the impact of operation technique without over-sewing of the staple line on the rate of postoperative complications. Reinforcement by over-sewing the staple line was mostly adopted to reduce postoperative bleeding and leak [13, 20, 21]. Omitting this step can in our experience shorten the operation time. In our technique, we wait 40-60 s after closing or firing the stapling device, respectively (twice three-row blue cartridge), to manage regular stapling and avoid bleeding or failure of the staple line, with no need to over-sew the staple line. No intraoperative leak test is currently performed, but the staple line is carefully inspected before ending of the procedure and any bleeding from the margin of the staple line is managed with single resorbable stitches. The number of postoperative complications in our set of patients operated without over-sewing of the staple line was very low and fully comparable to those reported by others using LSG with over-sewing of the staple line [20]. We therefore suggest that over-sewing of the staple could be omitted without compromising the safety of the procedure. 


\section{Conclusions}

The twin pandemic of obesity and type 2 diabetes represents one of the major health problems in the present century that influences many aspects of public health with consequences in psycho-social and socio-economic aspects. Bariatric surgery is an effective and powerful treatment for morbid obesity and obesity associated type 2 diabetes mellitus or other comorbidities. Laparoscopic sleeve gastrectomy is more frequently accepted as a stand-alone bariatric/ metabolic procedure, not only as a staged operation for high-risk morbidly obese patients. All parameters of metabolic syndrome with resolving/improving T2DM, hypertension, joint disease, depressive disorders or sleep apnoea are normalized or markedly improved after LSG. In case of non-satisfactory weight loss/metabolic improvement there is a possibility to convert LSG into duodenojejunal sleeve gastric bypass or biliopancreatic diversion/duodenal switch.

In our study we present 7-year experience with LSG on a large number of patients and stress the fact that over-sewing can be omitted while performing this procedure without compromising the outcomes and without increasing the number of postoperative complications. According to our experience, a carefully performed staple line in laparoscopic sleeve gastrectomy without over-sewing is feasible and safe. Our approach not only brings economic benefit by shortening the time of surgery roughly by $10 \mathrm{~min}$ (personal observation), but also decreases the time of anaesthesia, and last but not least excludes the possibility of excessive narrowing of the tubulised stomach. Furthermore, our data suggest that major sleeve restriction with $36 \mathrm{Fr}$ orogastric tube calibration has a superior weight-reducing effect compared to 42 Fr orogastric tube calibration.

\section{Acknowledgments}

Supported by RVO-VFN64165/2012; ZRO MO1012.

\section{References}

1. Ikramuddin S, Buchwald H. How bariatric and metabolic operations control metabolic syndrome. Br J Surg 2011; 98: 1339-41.

2. Hady HR, Dadan J, Luba M. The Influence of laparoscopic sleeve gastrectomy on metabolic syndrome parameters in obese patients in own material. Obes Surg 2012; 22: 13-22.

3. Buchwald H, Estok R, Fahrbach K, et al. Weight and type 2 diabetes after bariatric surgery: systematic review and meta-analysis. Am J Med 2009; 122: 248-56.
4. Hess DS, Hess DW. Biliopancreatic diversion with a duodenal switch. Obes Surg 1988; 8: 267-82.

5. Johnson D, Dachtler J, Sue-Ling HM, et al. The Magenstrasse and Mill operation for morbid obesity. Obes Surg 2003; 13: 10-6.

6. Weiner RA, Weiner S, Pomhoff I, et al. Laparoscopic sleeve gastrectomy - influence of sleeve size and resected gastric volume. Obes Surg 2007; 17: 1297-305.

7. Bobowicz M, Lehmann A, Orlowski M, et al. Prelimitary outcomes 1 year after laparoscopic sleeve gastrectomy based on bariatric analysis and reporting outcomes system (BSAROS). Obes Surg 2011; 21: 1843-8.

8. Kasalicky M, Michalsky D, Housova J, et al. Laparoscopic sleeve gastrectomy without an over-sewing of the staple line. Obes Surg 2008; 18: 1257-62.

9. Gumbs AA, Gagner M, Dakin G, et al. Sleeve gastrectomy for morbid obesity. Obes Surg 2007; 17: 962-9.

10. Selecchia G, Boru C, Pecchia A, et al. Effectivness of laparoscopic sleeve gastrectomy (first stage of biliopancreatic diversion with duodenal switch) on co-morbities in super-obese high-risk patients. Obes Surg 2006; 16: 1138-44.

11. Nocca D, Guillaume F, Noel P, et al. Impact of laparoscopic sleeve gastrectomy and laparoscopic gastric bypass on HbAlc blood level and pharmacological treatment of type 2 diabetes mellitus in severe or morbidly obese patients. Results of a multicenter prospective study at 1 year. Obes Surg 2011; 21: 738-43.

12. Fried M, Hainer V, Basdevand A, et al. Interdisciplinary European guidelines for surgery of severe (morbid) obesity. Obes Surg 2007; 17: 260-70.

13. Basso N, Casella G, Rizzello M. Laparoscopic sleeve gastrectomy as first stage or definitive intent in 300 conscutive cases. Surg Endosc 2011; 25: 444-9.

14. Melissas J, Daskalakis M, Koukouraki S, et al. Sleeve gastrectomy - a "food limiting" operation. Obes Surg 2008; 18: 1251-6.

15. Langer FB, Reza Hoda MA, Bohdjalian A, et al. Sleeve gastrectomy and gastric banding: effects on plasma ghrelin levels. Obes Surg 2005; 15: 1024-9.

16. Hady RH, Dadan J, Golaszewski P, Safiejko K. Impact of laparoscopic sleeve gastrectomy on body mass index, ghrelin, insulin and lipid levels in 100 obese patients. Videosurgery Miniinv 2012; 7: 251-9.

17. Vidal J, Ibarzabal A, Nicolau J, et al. Short-term effects of sleeve gastrectomy on type 2 diabetes mellitus in severely obese subjects. Obes Surg 2007; 17: 1069-74.

18. Himpens J, Dapri G, Cadière GB. A prospective randomized study between laparoscopic gastric banding and laparoscopic isolated sleeve gastrectomy: result after 1 and 3 years. Obes Surg 2006; 16: 1450-6.

19. Kasalicky M, Michalsky D, Housova J, et al. Laparoscopic gastric tubulisation - sleeve gastrectomy - another option for bariatric food intake restriction in morbidly obese subjects. Rozhl Chir 2007; 86: 601-6.

20. Chen B, Kiriakopoulos A, Tsakayannis D. Reinforcement does not necessarily reduce the rate of staple line leaks after sleeve gastrectomy. A review of the literature and clinical experiences. Obes Surg 2009; 19: 166-72.

21. Szewczyk T, Janczak P, Duszewski M, Modzelewski B. Technical problems in laparoscopic sleeve gastrectomy. Videosurgery Miniinv 2009; 4: 95-101. 
22. Patrikakos P, Toutouzas KG, Gazouli M, et al. Long-term plasma ghrelin an leptin modulation after sleeve gastrectomy in wistar rats in comparison with gastric tissue ghrelin expression. Obes Surg 2011; 21: 1432-7.

23. Murr M, Rafiei A, Habib A, Tannous KF. Overview of emerging concepts in metabolic surgery. Permanent J 2010; 14: 57-62.

24. Scopinaro N. Metabolic surgery. A new surgical discipline? J Med Sci 2010; 3: 28-34.

25. Buzga M, Holeczy P, Svagera Z, et al. Effects of sleeve gastrec tomy on parameters of lipid and glucose metabolism in obese women -6 months after operation. Videosurgery Miniinv 2013; 8: 22-8.

26. Basso N, Capoccia D, Rizzello F, et al. First-phase insulin secretion, insulin sensitivity, ghrelin, GLP-1, and PYY chages $72 \mathrm{~h}$ after sleeve gastrectomy in obese diabetic patients: the gastric hypothesis. Surg Endosc 2011; 25: 3540-50.

Received: 24.05.2013, accepted: 23.06.2013. 